\title{
The effects of dietary fructooligosaccharide on growth, intestinal short chain fatty acids level and hepatopancreatic condition of the giant freshwater prawn (Macrobrachium rosenbergii) post-larvae
}

\begin{abstract}
Prebiotics are one of the feed additives being studied to potentially enhance the growth of aquatic animals. The effects of dietary fructooligosaccharide (FOS) additives at $0.1 \%, 0.4 \%$, $1 \%$ and $2 \%$ on the growth performance, superoxide dismutase (SOD), lipid peroxidation, intestinal short chain fatty acids (SCFA) and hepatopancreatic histopathology of the freshwater prawn Macrobrachium rosenbergii post-larvae (initial weight \pm SE of $22.8 \pm 0.2$ $\mathrm{mg}$ ) were evaluated after 56 days of feeding. Each aquarium contained 80 post-larvae and each treatment was quadruplicated which yielded 320 prawns/treatment. The results showed that the specific growth rate for length and weight of the prawns was highest in the $0.4 \%$ dietary FOS treatment compared to all others. Prawns fed the $0.4 \%$ FOS diet had significantly higher $(\mathrm{P}<0.05)$ intestinal acetic acid than the control or $0.1 \%$ FOS treatments as well as significantly higher propionic acid than all other treatments. The amount of butyric acid was similar among all the dietary FOS treatments $(\mathrm{P}>0.05)$. Increasing dietary FOS significantly increased lipid peroxidation and decreased SOD inhibition rate (\%), indicating oxidative stress to the prawns. On day 28 , prawns fed 0.1 or $0.4 \%$ FOS had significantly more E-cells within their hepatopancreatic tubules compared to other treatments while after 56 days, the hepatopancreatic tubules of prawns in the $0.4 \%$ FOS treatment were more closely arranged with significantly more R- and E-cells. In some instances, prawns fed the 1 or $2 \%$ FOS diets had hypertrophied B-cells. The results demonstrate that the optimal tested FOS level to M. rosenbergii post-larvae was $0.4 \%$, and higher levels of 1 or $2 \%$ dietary FOS induced oxidative stress and reduced their hepatopancreatic condition.
\end{abstract}

Keyword: Short chain fatty acids; Prebiotic; Lipid peroxidation; Histology; Oxidative stress; Freshwater prawn 